Trauma Berufskrankh 2018 20 (Suppl 4):S260-S264 https://doi.org/10.1007/s10039-018-0373-z Online publiziert: 17. Mai 2018

(c) Springer Medizin Verlag GmbH, ein Teil von Springer Nature 2018

CrossMark

J. Zaage $\cdot$ M. Heinke

BG Klinikum Bergmannstrost Halle gGmbH, Halle, Deutschland

\title{
Zeitgemäßes Belegungs- und Entlassungsmanagement
}

- keine oder zu späte Information der Angehörigen,

- schlechte Erreichbarkeit eines kompetenten Ansprechpartners im Krankenhaus bei Rückfragen,

- keine zeitnahe Information des Unfallversicherungsträgers (der Entlassungsbrief traf in $72 \%$ der Fälle erst 2 Wochen oder noch später ein) [5].

\section{Maßnahmen}

\section{Hintergrund/Ausgangslage}

Niedergelassene D(Durchgangs)-Ärzte, Hausärzte, BG(Berufsgenossenschaft)Verwaltungen, ambulante Pflegedienste und auch Patienten klagen über Kommunikationsdefizite bei Entlassung der Patienten aus dem Krankenhaus [3-5, 7, 8].

Insbesondere werden bemängelt:

- kein Entlassungsbrief bei ambulanter Vorstellung,

- unvollständige Inhalte des Entlassungsbriefes,

- mangelnde Angaben zur Medikationsveränderung während des stationären Aufenthaltes bzw. zu den Medikationsempfehlungen nach dem stationären Aufenthalt,

- kurzfristige Entlassung aus dem Krankenhaus ohne Information des weiterbehandelnden Arztes,

- fehlende Absprache über ärztliche, pflegerische und rehabilitative Maßnahmen, unklare Verantwortlichkeit zur Patientenkoordination nach stationärer Behandlung,

- mangelnde Vorbereitung einer Entlassung am Freitagnachmittag,

- keine oder zu späte Information des ambulanten Pflegedienstes,
Vor ca. 10 Jahren haben wir am Bergmannstrost neben den Fortbildungsveranstaltungen für D-Ärzte auch eine jährliche Fortbildungsreihe „Unser gemeinsamer Patient" mit den Hauptzuweisern begonnen, die sich inzwischen fest etabliert hat. Außerdem haben wir auf Anregung unseres Geschäftsführers ein zentrales Belegungs- und Entlassungsmanagement etabliert.

\section{Fortbildungsreihe "Unser gemeinsamer Patient ${ }^{\prime \prime}$}

Um die teils mangelhafte Kommunikation zwischen ambulantem und stationärem Sektor zu verbessern, haben wir von Anfang an neben fachlichen Themen auch immer wieder Schnittstellenprobleme besprochen. So war schon $2007 \mathrm{der}$ Arztbrief ein zentrales Thema, ab 2008 haben wir auch ambulante Pflegedienste eingebunden.

Im Jahr 2017 haben wir die Veröffentlichung des Rahmenvertrages über ein Entlassmanagement nach $₫ 39$ SGB V [6] thematisiert. Da wir die Veranstaltungsreihe jährlich evaluieren, können wir nach 10-jähriger Erfahrung konstatieren, dass sich die Kommunikation zwischen niedergelassenen Ärzten und dem Krankenhaus erheblich verbessert hat und das Verständnis füreinander deutlich gewachsen ist.

\section{Zentrales Belegungs- und Entlassungsmanagement}

Grundsätzlich soll ein Belegungsmanagement sicherstellen, dass möglichst jeder Patient zur richtigen Zeit im richtigen Bett liegt [7]. Das gilt für alle geplanten (elektiven) wie auch möglichst alle ungeplanten (akuten) Aufnahmen. Damit wollen wir gleichzeitig sicherstellen, dass die vorhandenen Kapazitäten optimal genutzt werden $[3,8]$.

Das Entlassungsmanagement hat zum Ziel, dass die Patienten gut vorbereitet und ohne Verlust von Informationen (Entlassungsbrief, Verordnungen, Dokumente ...) nahtlos entweder im ambulanten Sektor oder in der stationären Pflege weiter betreut werden können. Damit handelt es sich in erster Linie um eine koordinierende Funktion.

\footnotetext{
Infobox 1 Faktoren bei stationärer Patientenaufnahme

- Indikation

- Diagnostik vollständig?

- Terminierung Operation (Kapazität, Personal)

- Terminierung Intervention (Kapazität, Personal)

- Bett

- Aktuelle Lebenssituation des Patienten beeinflusst die Entlassung: häusliches Umfeld, geistige/körperliche Defizite, Medikamente

- Verweildauer
} 


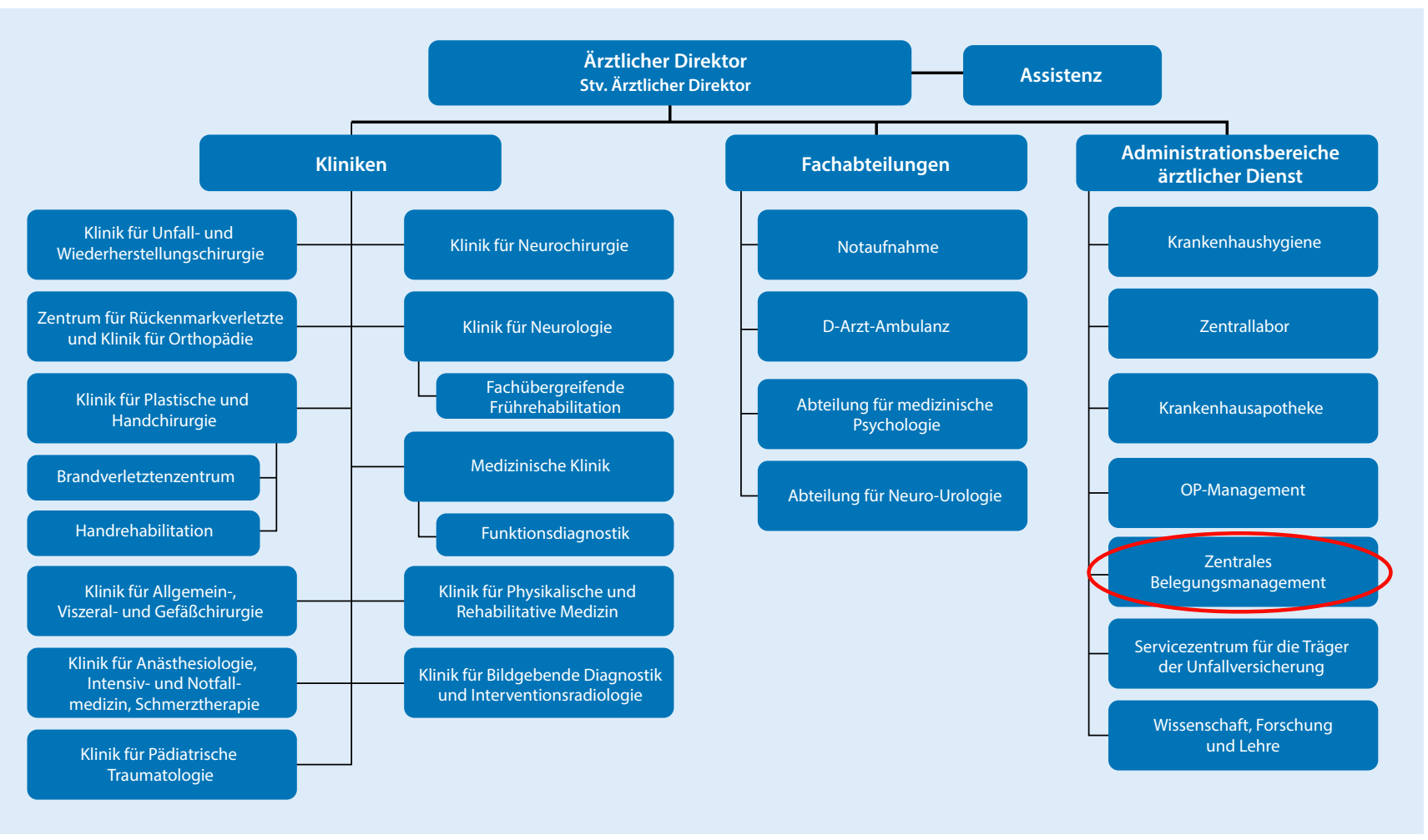

Abb. 1 A Verantwortungsbereich des Ärztlichen Direktors

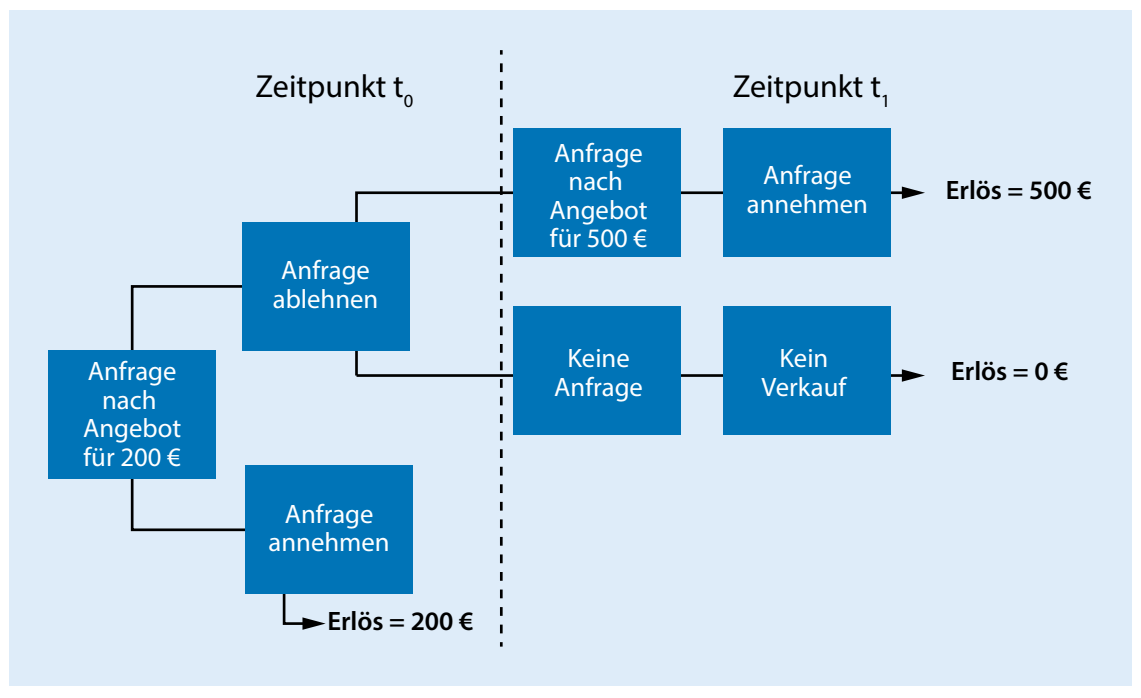

Abb. 2 ^ Mögliches Erlösszenario. (Aus [8])

\section{Belegungsmanagement}

Formal untersteht das Belegungsmanagement (wie auch das Operationsmanagement) als Administrationsbereich dem Ärztlichen Direktor (• Abb. 1). Dies ist notwendig, damit in einer möglichen strittigen Situation die Interessen des Krankenhauses gegenüber denen der einzelnen Fachabteilungen entsprechend vertreten werden können.

Entscheidungen des Belegungsmanagements können auch teils erhebliche ökonomische Auswirkungen auf das Krankenhaus haben. Dies soll • Abb. 2 als abstraktes Beispiel (aus [8]) verdeutlichen.

Um diesen Umstand immer wieder mit zu berücksichtigen und nicht ganz aus dem Blick zu verlieren, setzt sich unsere wöchentliche Besprechungsrunde aus folgenden Bereichen zusammen, wobei jeweils eine Mitarbeiterin bzw. ein Mitarbeiter vertreten ist:

- Belegungsmanagement (akut und Reha),

- ärztliche Direktion,

- Pflegedirektion,

- Sozialdienst/Entlassungsmanagement,

- Controlling und Finanzen,

- Servicebüro für die Unfallversicherer,

- Patientenaufnahme,

- Medizincontrolling.

In dieser Konstellation tagen wir wöchentlich und besprechen die anstehenden Probleme. Über die Sitzung wird ein Protokoll gefertigt, das auch der Geschäftsführung zur Verfügung gestellt wird.

Hauptamtlich arbeiten 2 Mitarbeiterinnen für das Belegungsmanagement, alle anderen sind nebenamtlich tätig.

Die täglichen Belegungen steuern im Wesentlichen 4 Mitarbeiterinnen bzw. Mitarbeiter - die beiden hauptamtlichen und 2 nebenamtliche. In der Literatur [3] kann man lesen: 
„Die informationstechnologische Umsetzung kann über das vorhandene Krankenhausinformationssystem oder eine hierfür spezialisierte Software erfolgen."

In der Praxis stellt sich das allerdings schwierig dar, da je nach Zielstellung verschiedene statische oder dynamische Statistiken im System zu finden sind. Wenn beispielsweise ein Neurologe aus der Notaufnahme ein Bett sucht, so stellt er fest, dass auf seiner eigenen Station bereits ein Zusatzbett aufgestellt ist. In der Neurochirurgie dagegen ( $\bullet$ Abb. 3 ) vermutet er freie Betten und ist überrascht, dass diese effektiv nicht zur Verfügung stehen. Bei Anruf teilt ihm die Station mit, dass ein Zimmer renoviert wird und einige Zimmer wegen besonderer Keime (z.B. Methicillin-resistenter Staphylococcus aureus [MRSA]) und entsprechender Isolierungsmaßnahmen nicht voll belegt werden können. All diese Dinge gehen aus den allgemeinen Statistiken nicht hervor. Die einzelnen Stationsgrafiken dagegen würden diese Umstände erkennen lassen, aber darauf hat z. B. der entsprechende Neurologe datenschutzrechtlich keinen Zugriff.

Aus diesen ganz praktischen Gründen rufen inzwischen alle Mitarbeiterinnen und Mitarbeiter in entsprechenden Situationen direkt beim Belegungsmanagement an, um ein adäquates Bett für einen entsprechenden Patienten zu bekommen.

Wir halten zurzeit im Bergmannstrost 10 Fachabteilungen vor und haben deshalb eine Kombination von zentraler und dezentraler Steuerung etabliert. Ein zentraler Belegungsmanager kann weder alle Dienst- und Urlaubspläne noch die Krankheitsvertretungen in allen Fachabteilungen kennen. Außerdem überblickt er auch nicht die Fachexpertise der gesamten Ärzteschaft. Plant z.B. ein Unfallchirurg einen komplexen Eingriff, der die Anwesenheit eines bestimmten Operateurs nötig macht, so fordert er (dezentral) zu einer ganz bestimmten Zeit ein Bett an. Das Belegungsmanagement (zentral) setzt die Anforderung um und garantiert intern zum entsprechenden Zeitpunkt das Bett (in solch komplexen Fällen in Abstimmung mit dem Operationsmanagement).

Trauma Berufskrankh 2018 · 20 (Suppl 4):S260-S264

https://doi.org/10.1007/s10039-018-0373-z

(c) Springer Medizin Verlag GmbH, ein Teil von Springer Nature 2018

\section{J. Zaage $\cdot$ M. Heinke}

\section{Zeitgemäßes Belegungs- und Entlassungsmanagement}

\section{Zusammenfassung}

Während ein zeitgemäßes Entlassungsmanagement zentral gesteuert werden sollte, kann das Belegungsmanagement ebenfalls zentral, aber auch in einer Kombination mit zentralen und dezentralen Mechanismen gesteuert werden. Wesentlichen Einfluss darauf haben die Größe (Bettenzahl) und Struktur (Zahl der Fachabteilungen) des jeweiligen Krankenhauses. Bei nur einer oder wenigen Fachabteilungen bietet sich die zentrale Steuerung an, bei vielen Fachabteilungen eher die Kombination aus zentraler und dezentraler Steuerung. Weitere Einflussfaktoren sind die Patientenstrukturen, wie das Verhältnis zwischen berufsgenossenschaftlich, gesetzlich und privat Versicherten und das Verhältnis von
Akut- zu Elektivaufnahmen. Zusätzlich sind zu berücksichtigen: die Rate der stationären Wiederaufnahmen, komplexe Fallkonstellationen, Intensivmedizin/Beatmungs-DRGs, Keimbesiedlungen/Infektionen, Mutter/Kind, Patient/Angehörige, Budget- und Entgeltverhandlung. Unter Beachtung der genannten Einflussfaktoren ist ein zeitgemäßes Belegungs- und Entlassungsmanagement sehr stark geprägt von Verantwortung in dreierlei Hinsicht: 1. medizinisch/fachlich/pflegerisch, 2. organisatorisch und 3. ökonomisch.

\section{Schlüsselwörter}

Bettenzahl · Fachabteilungen · Zentrale

Steuerung · Dezentrale Steuerung ·

Patientenstrukturen

\section{Modern hospital admission and discharge management}

\section{Abstract}

A modern discharge management should be centrally organized but patient allocation management can also be centrally organized in addition to a combination of centralized and decentralized mechanisms. This is substantially influenced by the size (number of beds) and structure (number of specialist departments) of each hospital. With only one or only a few specialist departments centralized organization is the best solution but with many specialist departments a combination of centralized and decentralized management is more appropriate. Further influencing factors are patient structures, such as the relationship between occupational insurance shemes, statutory health insurance and private health insurance as well as the ratio of elective and emergency admissions. In addition, the following factors must be taken into consideration: the rate of inpatient rehospitalization, complex case constellations, intensive care medicine and ventilation diagnosis-related groups (DRG), pathogen colonization/infections, mother/child, patient/relatives and dealing with budgets and remuneration. Taking these influencing factors into consideration a modern hospital admission and discharge management is greatly influenced by responsibility in three respects: 1 . medical, specialized, nursing, 2. organizational and 3. economic areas.

\section{Keywords}

Number of beds - Specialist departments . Centralized management - Decentralized management $\cdot$ Patient structures
Auch Patientenübernahmen von auswärtigen Krankenhäusern verlangen immer Absprachen zwischen dezentraler Fachabteilung und zentralem Belegungsmanagement, um einen reibungslosen Ablauf zu garantieren.

Bei der Planung einer stationären Aufnahme sind mehrere Gesichtspunkte zu berücksichtigen (-Infobox 1). Die Indikation wird rein ärztlich gestellt, bei allen anderen Punkten wird das Belegungsma- nagement organisatorisch und beratend tätig.

Die Verweildauer ist im heutigen $\mathrm{Ab}$ rechnungssystem von zentraler Bedeutung. Der Arzt kann bei seiner Entscheidung zur Entlassung des Patienten auf vielfältige Weise vom Belegungsmanagement unterstützt werden (•Infobox 2). 


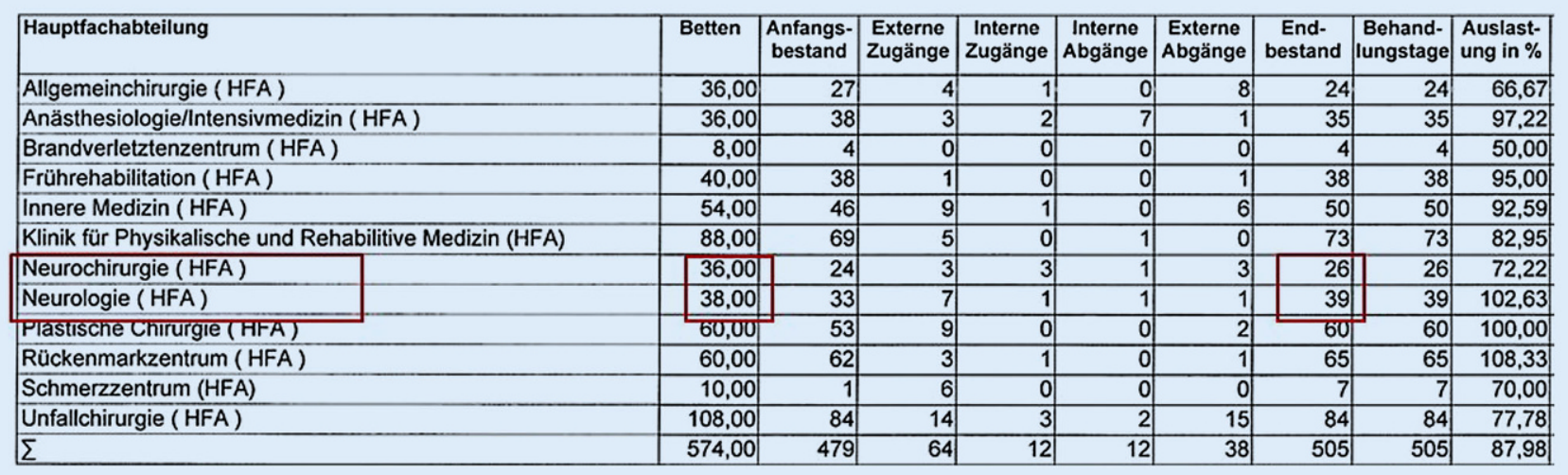

Abb. 3 ^ Auszug aus der "Mitternachtsstatistik"

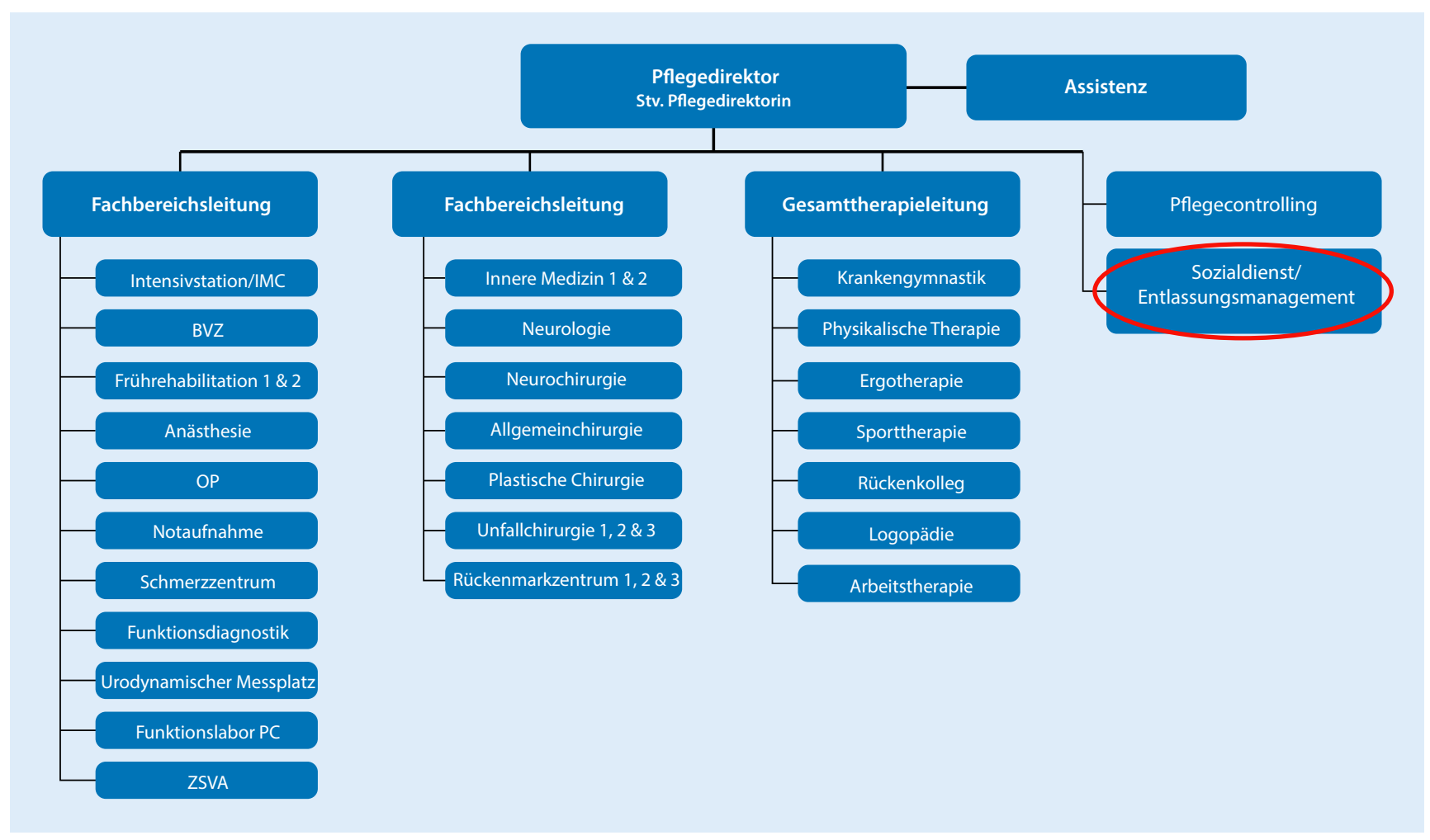

Abb. 4 A Verantwortungsbereich des Pflegedirektors. BVZ Brandverletztenzentrum, ZSVA Zentrale Sterilgutversorgungsabteilung

\section{Entlassungsmanagement}

Formal untersteht das Entlassungsmanagement im Gegensatz zum Belegungsmanagement der Pflegedirektion (• Abb. 4). Das hat bei uns rein historische Gründe, da der Sozialdienst schon vor Einführung eines Belegungsmanagements so organisiert war - mit der Zuständigkeit für die Entlassungen.

In der Praxis funktioniert das $\mathrm{Zu}$ sammenspiel von Belegungs- und Entlas- sungsmanagement reibungs- und nahtlos, und zwar sowohl in der Tagesroutine als auch bei prinzipiellen Fragen.

Eine Entlassung aus dem Krankenhaus sollte gut vorbereitet sein, der Zeitpunkt rechtzeitig abgestimmt sein und die Situation nach der Entlassung geklärt sein. Gerade hier werden die meisten Mängel beklagt - und das auch im SGB VII-Bereich, obwohl die Voraussetzungen optimal sind ([3], S. 71). Deshalb gehören zu den Hauptaufgaben im Entlassungsmanagement:

- die Ermittlung des patientenseitigen Bedarfs,

- die Erstellung eines Entlassplanes,

- die Aufklärung des Patienten über die Versorgung nach Entlassung,

- der Informationsaustausch mit ambulanten Leistungserbringern,

- die Verordnung von ambulanten Leistungen. 


\section{Infobox 2 Beeinflussung der Verweildauer (Verweildauer- „Management") \\ - Entscheidung des Arztes \\ - Flankierende Unterstützung \\ - Koordinierung \\ - Dokumentation der Leistungen \\ - MDK(Medizinischer Dienst der Kranken- versicherung)-konforme Dokumentation \\ - Erlöse/DRG \\ - Teamarbeit \\ - Transparenz}

Um die Entlassung bereits von der Aufnahme an im Blick zu haben, hängt in allen Sekretariaten und Arztzimmern auf den Stationen eine von uns selbst entwickelte Checkliste mit allen möglicherweise zu berücksichtigenden Punkten (•Infobox 3 ).

Im Netz haben wir einen selbst erstellten Entlassplan (elektronisches Zusatzmaterial online, ESM-Abb. 1), den wir so überarbeitet haben, dass er die Kriterien nach Rahmenvertrag $\$ 39[1,2,6]$ erfüllt. Dieser Entlassplan kann dann bei Bedarf fortlaufend bei jeder Visite immer weiter vervollständigt werden und bleibt als Dokument in der elektronischen Krankenakte.

Unter Berücksichtigung des Entlassplanes erhält jeder Patient am Entlassungstag den Entlassungsbrief und - falls nötig - die entsprechenden Verordnungen. Die Medikation aus dem Entlassungsbrief wird automatisch in einen zusätzlich erstellten Medikationsplan übernommen und dem Patienten ebenfalls ausgehändigt. Mit dieser EDV-seitigen Verknüpfung von Entlassungsbrief und Medikationsplan sind widersprüchliche Angaben, die früher durchaus ein Problem waren, jetzt ausgeschlossen.

Mit all diesen Maßnahmen und unter erheblichem Aufwand ist ein zeitgemäßes und verantwortungsvolles Belegungs- und Entlassungsmanagement möglich und entspricht in großen Teilen einem Fallmanagement.

\section{Fazit}

Während ein zeitgemäßes Entlassungsmanagement nach unserer Erfahrung immer zentral gesteuert werden sollte, kann das Belegungsmanagement sowohl zentral als auch in einer Kombination

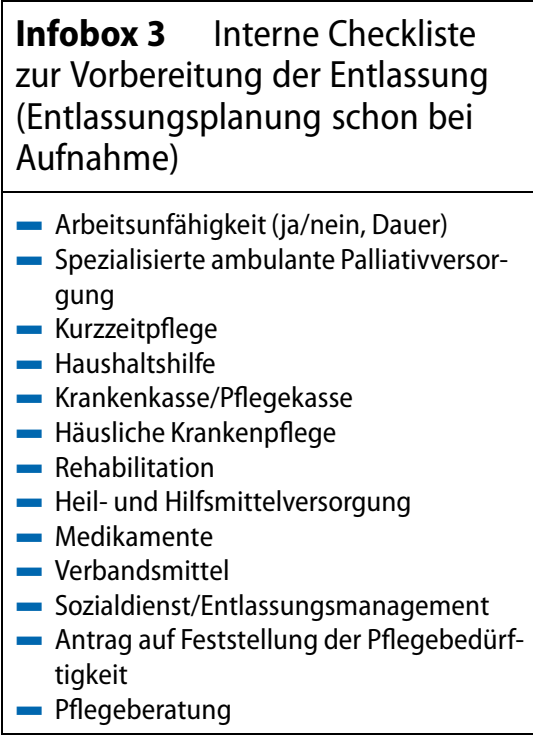

aus zentralen und dezentralen Elementen gesteuert werden. Bei nur einer oder wenigen Fachabteilungen bietet sich die zentrale Steuerung an, ansonsten eher die Kombinationsvariante. Weitere wesentliche Einflussfaktoren sind die Patientenstrukturen, das Verhältnis von Akut- zu Elektivaufnahmen, die Rate der stationären Wiederaufnahmen und komplexe Fallkonstellationen. Somit ist ein zeitgemäßes Belegungs- und Entlassungsmanagement geprägt von - medizinisch/fachlich/pflegerischer, - organisatorischer und letztlich auch - ökonomischer Verantwortung.

\section{Korrespondenzadresse}

\section{Heinke}

BG Klinikum Bergmannstrost Halle gGmbH

Merseburger Str. 165, 06112 Halle, Deutschland Marion.Heinke@bergmannstrost.de

\section{Einhaltung ethischer Richtlinien}

Interessenkonflikt. J. Zaage und M. Heinke geben an, dass kein Interessenkonflikt besteht.

Dieser Beitrag beinhaltet keine von den Autoren durchgeführten Studien an Menschen oder Tieren.

The supplement containing this article is not sponsored by industry.

\section{Literatur}

1. Aktualisierte und überarbeitete Umsetzungshinweise der DKG zum Rahmenvertrag über ein Entlassmanagement beim Übergang in die Versorgung nach Krankenhausbehandlung nach $\S 39$ Abs. 1a S. 9 SGB V. Krankenhausgesellschaft Sachsen-Anhalt Mitteilung Nr. 421/2017

2. Baum G (2016) Entlassmanagement im dreiseitigen Hürdenlauf. Krankenhaus 108(5):365

3. Deimel D, Müller M-L (2013) Entlassmanagement. Thieme, Stuttgart, New York

4. Hoffmann F, Ohmann T, Hax P-M (2016) Entlassmanagement im BGlichen Heilverfahren. Trauma Berufskr 18(4):331-334

5. Polak U (2017) Wie zufriedenstellend ist die Zusammenarbeit mit den SAV-Kliniken? DGUV Forum 9(9):26-28

6. Rahmenvertrag über ein Entlassmanagement beim Übergang in die Versorgung nach Krankenhausbehandlung nach $\S 39$ Abs. 1a S. 9 SGB V (Rahmenvertrag Entlassmanagement)

7. Schmidt R, Pukall I, Köhler M (2017) Zur richtigen Zeit im richtigen Bett? Heilberufe 69(3):48-50

8. Zapp W (2014) Strategische Entwicklung im Krankenhaus Kennzahlen-Portfolio GeokodierungBelegungsmanagement. Kohlhammer, Stuttgart 